\title{
Outcome measures in asthma
}

\author{
N C Barnes \\ Department of Respiratory Medicine, The London Chest Hospital, London E2 9JX, \\ UK
}

Introductory articles

\section{Exacerbations of asthma: a descriptive study of 425 exacerbations}

A E Tattersfield, D S Postma, P J Barnes, K Svensson, C-A Bauer, P M O’Byrne, C-G Lofdahl, R A Pauwels, A Ullman on behalf of the FACET International Study Group

The identification, prevention, and prompt treatment of exacerbations are major objectives of asthma $\overrightarrow{\dot{c}}$ management. We looked at change in PEF, symptoms, and use of rescue $\beta$ agonists during the 4250 severe exacerbations that occurred during a 12-month parallel group study (FACET) in which low and $\bigcirc$ high doses of budesonide with and without formoterol were compared in patients with asthma. Oral $\vec{\longrightarrow}$ corticosteroids were prescribed for severe exacerbations, the main study end point, defined as the need of for a course of oral corticosteroids $(n=311)$ or a reduction in morning PEF of $>30 \%$ on two consecutive days, PEF, symptoms, and bronchodilator use over the 14 days before and after the exacerbation were $\tilde{O}$ obtained from diary cards. Exacerbations were characterized by a gradual fall in PEF over several days,. followed by more rapid changes over 2-3 days: an increase in symptoms and rescue $\beta$ agonist use 8 occurred in parallel, and both the severity and time course of the changes were similar in all treatment groups. Exacerbations identified by the need for oral corticosteroids were associated with more symptoms and smaller changes in PEF than those identified on the basis of PEF criteria. Female sex was $\frac{0}{0}$ the main patient characteristic associated with an increased risk of having a severe exacerbation. $\stackrel{2}{\vec{P}}$ Exacerbations may be characterized predominantly by change in symptoms or change in PEF, but the $\frac{0}{3}$ pattern was not affected by the dose of inhaled corticosteroid or by whether the patient was taking formoterol. (Am J Respir Crit Care Med 1999;160:594-9)

\section{Differences between asthma exacerbations and poor asthma control}

\section{H Reddel, S Ware, G Marks, C Salome, C Jenkins, A Woolcock}

Background. Increased variation in peak expiratory flow (PEF) is characteristic of poorly controlled asthma and measurement of diurnal variability of PEF has been recommended for assessment of asthma severity, including during exacerbations. We aimed to test whether asthma exacerbations had the same PEF characteristics as poor asthma control. Methods. Electronic PEF records from 43 patients with initially poorly controlled asthma were examined for all exacerbations that occurred after PEF reached $\%$

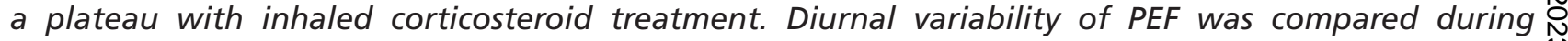
exacerbations, run-in (poor asthma control), and the period of stable asthma before each exacerbation. Findings. Diurnal variability was $21.3 \%$ during poor asthma control and improved to $5.3 \%$ (stable asthma) with inhaled corticosteroid treatment. 40 exacerbations occurred in 26 patients over 2-16 months; 38 (95\%) of exacerbations were associated with symptoms of clinical respiratory infection. During exacerbations, consecutive PEF values fell linearly over several days then improved linearly. $\frac{0}{0}$ However, diurnal variability during exacerbations (7.7\%) was not significantly higher than during stable $\underset{\otimes}{\circ}$ asthma $(5.4 \%, p=0.1)$. PEF data were consistent with impaired response to inhaled $\beta_{2}$ agonist during $\underset{\mathbb{Q}}{\mathbb{Q}}$ exacerbations but not during poorly controlled asthma. Interpretation. Asthmatics remain vulnerable g to exacerbations during clinical respiratory infections, even after asthma is brought under control. 8 Calculation of diurnal variability may fail to detect important changes in lung function. PEF variation $\frac{8}{0}$ is strikingly different during exacerbations compared with poor asthma control, suggesting differences in $\beta_{2}$ adrenoceptor function between these conditions. (Lancet 1999;353:364-9) 
Table 1 Aims of asthma management: GINA guidelines 1993

- Minimal (ideally no) chronic symptoms, including nocturnal symptoms

- Minimal (infrequent exacerbations)

- No emergency visits

- Minimal (ideally no) need for as needed $\beta_{2}$ agonists

- No limitations on activities, including exercise

- Peak expiratory flow (PEF) diurnal variation of less than $20 \%$

- (Near) normal PEF

- Minimal (or no) adverse effects from medicine

Table 2 Aims of asthma management: GINA guidelines 1999

- Prevent troublesome symptoms night and day

- Prevent serious attacks

- Require little or no quick relief medication

- Have productive, physically active lives

- Have (near) normal lung function

One of the strengths of asthma guidelines is that they set out the aims of asthma management. These aims help clinicians, but are also increasingly influential in shaping the design of clinical trials by determining outcome measures. Current asthma guidelines emphasise the need for long term control of asthma. Asthma control is not defined by a single measurement such as normal respiratory function but by a whole series of parameters. The 1993 Global Initiative for Asthma (GINA) guidelines described seven efficacy aims plus emphasising the need for drug safety (table 1).3 These aims of asthma management can be broadly grouped into three areas: prevention of symptoms including exercise induced symptoms and minimal requirement for symptomatic relief with a short acting $\beta_{2}$ agonist; normalisation of lung function; and prevention of asthma exacerbations. The most recent version of the GINA guidelines has simplified these aims (table 2). The British Thoracic Society (BTS) guidelines also list the aims of asthma management (table 3)

To achieve these aims of asthma management the guidelines propose treatment often using a combination of different drugs. The guidelines add that this should be achieved with medications which have as few side effects as possible. Clinicians have to choose the drug or drugs they believe will achieve the aims of asthma management and asthma control. It is, however, becoming increasingly apparent that the choice of which drug or drugs to use is not as easy as it might outwardly appear and that the interrelationship between the different facets of asthma control such as symptoms, pulmonary function, and exacerbations is complex.

Prevention of asthma exacerbations is a very important aim of asthma management as exacerbations are disruptive for patients and costly to treat. However,

Table 3 Aims of asthma management: BTS guidelines 1993

- To recognise asthma

- To abolish symptoms

- To restore normal or best possible long term airway function

- To reduce the risk of severe attack

- To enable normal growth to occur in children

- To minimise absence from school or work while health care professionals and patients may be able to recognise an exacerbation, defining what an exacerbation is for the purpose of clinical trials is not easy. Individual patients and physicians may have a different propensity threshold for determining that a worsening of asthma is an exacerbation rather than what $\overrightarrow{\vec{c}}$ they consider is the normal inherent variability in the $\overrightarrow{0}$ condition. Furthermore, an individual's readiness to $\frac{\bar{\sigma}}{\sigma}$ report an exacerbation may vary over time because of $\overline{\bar{c}}$ changes in perception of symptoms related to changes $\stackrel{\vec{Q}}{2}$ in anxiety, depression or stress.

If a clinical outcome cannot be defined and measured it is difficult to determine reliably what effect drugs may have on this and to come to rational therapeutic choices. The situation is further confused by recent evidence that cherished notions of the interrelationship between, for instance, peak flow variability and exacerbations of asthma may not in fact be correct.

\section{What are asthma exacerbations?}

At present there is no universally accepted definition of an asthma exacerbation. Expert reports on asthma is outcomes have not addressed the issue directl ${ }^{5}$ or have only provided guidance 6

An exacerbation could be described as a worsening $\vec{P}$ of asthma that exceeds the normal day to day variability of lung function and symptoms and triggers, or should $\frac{\omega}{\omega}$ trigger, the need for increased treatment with more $\stackrel{\sim}{N}$ than just inhaled $\beta_{2}$ agonists. Measuring the number 8 of exacerbations has become an important outcome? measure in clinical trials. One of the first and the largest trials to measure asthma exacerbations prospectively was the FACET study. This 12 month long parallel group design study investigated four different treatment strategies: budesonide $100 \mu \mathrm{g}$ twice daily, budesonide $\stackrel{\mathbb{Q}}{\varrho}$ $100 \mu \mathrm{g}$ twice daily plus formoterol $12 \mu \mathrm{g}$ twice daily, $\overrightarrow{\overrightarrow{0}}$ budesonide $400 \mu \mathrm{g}$ twice daily, and budesonide $400 \mu \mathrm{g}$ twice daily plus formoterol $12 \mu \mathrm{g}$ twice daily. The primary outcome variable in the study was severe ex-? acerbations. The definition of a severe exacerbation was either requirement for a course of oral corticosteroids $\frac{0}{2}$ as judged by the clinical investigator or an episode in $\underset{x}{x}$ which morning peak expiratory flow $(\mathrm{PEF})$ fell by more 3 than $30 \%$ on two consecutive days from the mean morning PEF during the 10 days of the run in period. 3 852 patients entered the randomisation phase of the 0 study and 694 completed the one year long study. In this time there were 425 severe exacerbations. Patients kept daily diary cards in which they measured morning and evening PEF and scored their symptoms and use 5 of rescue medication; the study thus provided a unique $\mathrm{N}$ opportunity to investigate exacerbations and their re- N lation to PEF and symptoms ${ }^{8}$ The first interesting result from the further analysis study is that $70 \%$ of the 0 exacerbations were defined by a requirement for a course $\bar{\varnothing}$ of oral corticosteroids rather than by a $30 \%$ decrease in ? PEF. This means that, despite clinicians having available 0 to them carefully measured PEF, they chose to treat the $\frac{\overrightarrow{0}}{\mathbb{D}}$ patient for an exacerbation when the PEF had not fallen by $30 \%$. This is even more revealing when the maximum decrease in PEF is compared in those whose exacerbation was defined by oral steroid use and those 8 defined by a fall in PEF. In those defined as showing an exacerbation by a fall in PEF, decrease in peak flow 흘 was approximately twice as large as those defined by symptoms. The clue to the reason for this discrepancy probably lies in examination of the asthma symptoms score. Despite having a smaller decrement in PEF, the group defined by their requirement for oral cortico- 
steroids had a greater increase in symptoms than those defined by a $30 \%$ fall in PEF. Is this because these patients had a greater propensity for reporting symptoms when their lung function declined or was it something about the cause of the exacerbation which led to a relative excess in symptoms? It might be possible to shed some light on this from examining the records of those patients who had more than one exacerbation to see if different exacerbations had a common pattern or not. The importance of symptoms in determining the patient's and doctor's response to a worsening of asthma is further reinforced by another analysis performed in the paper. Despite being clearly instructed to contact the trialist if there was a $30 \%$ decrease in their PEF, 76 patients neither contacted the trialist nor had a course of steroids and yet their asthma recovered - if anything, more rapidly than those who took the oral corticosteroids. Again there was a difference in the symptom scores when this group and those who took oral steroids were compared. Those patients who took a course of oral steroids had a greater increase in symptoms than those who did not. This again indicates that symptoms were a very important factor in deciding on a prescription for oral steroids. Concern has been expressed that certain drugs, in particular long acting $\beta_{2}$ agonists, may blunt the patient's ability to perceive an asthma exacerbation. However, there was no difference in the pattern of asthma exacerbations in the four limbs of the study with no evidence that the prescription of long acting $\beta_{2}$ agonists blunted perception. The change in symptoms and PEF seemed to go in parallel, so there was little that patients could do to predict that they were about to have an exacerbation and act to prevent it.

\section{Relation between exacerbations and peak expiratory flow variability}

Analysis of the FACET study exacerbation data indicates that the relation between changes in pulmonary function and exacerbations is complex and either varies between patients or in the same patient between exacerbations. Further evidence of this is provided by the paper by Reddel et ad. In this study 43 patients were followed for at least three months. At the beginning of the study the authors say that the patients had poorly controlled asthma based on their symptom frequency, night time awakening, and bronchodilator use. It does seem reasonable to state that these patients had poorly controlled asthma. They had asthma symptoms on a mean of 5.1 days per week, they were waking on 2.4 nights per week with asthma, and their mean morning PEF was only $63.5 \%$ of predicted. There was a marked improvement when patients were treated with inhaled corticosteroids with asthma symptoms decreasing to 1.2 days per week, night time wakening to only 0.1 nights per week, and mean morning PEF improving to $90.2 \%$ of predicted. An asthma exacerbation was defined as a fall in PEF for at least two days of at least two standard deviations below the mean morning pre-bronchodilator PEF for the previous four weeks; the end of an exacerbation was defined as recovery of the PEF to above this level for at least two days.

Forty three per cent of patients had an exacerbation of their asthma. The authors do not report whether there were patients who had a course of oral steroids but did not meet these pulmonary function criteria. The pattern of change in PEF did not seem significantly different from the FACET study analysis. The key finding of Reddel et al was that, although PEF worsened, there was no increase in the diurnal variation in PEF which was in marked contrast to the run in period of the study. During the run in period when patients had $\vec{乛}$ unstable asthma they had marked diurnal variation in PEF. The authors suggest that, whereas marked diurnal variability is a feature of poor asthma control, when an $\overrightarrow{\vec{*}}$ exacerbation occurs PEF variability is not a feature. $\overrightarrow{0}$ They state that the asthma exacerbations were largely $\frac{\bar{\sigma}}{\sigma}$ due to viral infections, but the evidence for this is not $\overline{\bar{p}}$ compelling. The evidence they give for viral aetiology $\frac{\mathbb{d}}{2}$ of exacerbations is that there were typical symptoms of a viral upper respiratory tract infection yet viral isolation was not performed. They claim that exacerbations were $\vec{\circ}$ unlikely to be caused by allergen exposure as asthma in $\overrightarrow{\vec{\omega}}$ South Wales is perennial rather than seasonal because $\stackrel{\sigma}{\sigma}$ of the high year round levels of house dust mite, but $\overrightarrow{\overrightarrow{5}}$ this ignores the possibility that other allergens may have caused the exacerbations and that other factors such as $\dot{x}$ air pollution or a combination of air pollution and $\mathrm{fr}$ allergen exposure may have led to the exacerbation. $\frac{\text { D }}{0}$ The authors suggest that the lack of diurnal variation 0 is due to downregulation of $\beta_{2}$ receptor function caused by the viral infection. However, it is also possible that is lack of diurnal variation is a feature of any exacerbation

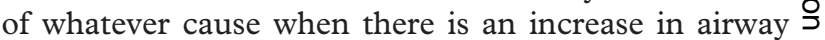
inflammation. Another possible explanation for the $\overrightarrow{\vec{t}}$ difference between the baseline period of poor asthma control and the exacerbation of asthma is that the use of inhaled corticosteroids modified the diurnal variation. During the baseline period only 12 of the 26 patients 8 who experienced at least one exacerbation had been taking inhaled steroids for three months or more. Patients were then started on regular treatment with the inhaled budesonide, although the dose of budesonide during the treatment stage is not reported. However, all patients were treated with inhaled steroids and pre- $\stackrel{\mathbb{Q}}{\varrho}$ sumably those who were on inhaled steroids during the $\overrightarrow{\overrightarrow{0}}$ run in phase had the inhaled steroid dose increased. It 3 is known that inhaled steroids reduce diurnal variability in PEF. It is therefore possible that, in the absence of inhaled corticosteroids, an asthma exacerbation may cause increased PEF but, in the presence of adequate $\frac{0}{2}$ doses of inhaled steroids, although there is a fall in $x$ peak flow, diurnal variability does not increase. This $\underline{3}$. interpretation is supported by a study in patients with mild asthma in whom exacerbations of asthma were 3 induced by inoculation with rhinovirus.$^{10}$ Lung function 0 was monitored with home spirometry and an increase in diurnal variation occurred.

These findings should give pause for thought and stimulate further research. They indicate that the re- of lationship between different asthma outcome measures $N$ is not simple and that these relationships are different $\underset{\omega}{N}$ in different patients or vary in the same patient across time. They show that apparently simple clinical concepts 0 such as what constitutes an asthma exacerbation or the variability in peak flow in relation to episodes of poor $\stackrel{\mathcal{\infty}}{?}$ asthma control are complex and ill understood. This has implications for clinical practice, research, and understandings of basic mechanisms.

\section{Implications for clinical practice}

The guidelines for asthma treatment clearly state that asthma control can only be defined by looking at a 흘 number of parameters (tables 1,2 and 3). The guidelines $\stackrel{?}{?}$ also indicate that in most patients a combination of drugs is needed to control asthma - for example, inhaled corticosteroids plus short acting $\beta_{2}$ agonists at step 2 of the BTS or GINA guidelines and inhaled corticosteroids 
and long acting bronchodilators or leukotriene receptor antagonists or theophyllines plus short acting $\beta_{2}$ agonists at step 3. Although they recognise the need for multiple drug therapy for many patients with asthma, they do not begin to help the practitioner in choosing between these different options nor do they articulate the fact that different drug therapies may have different relative strengths and weaknesses when it comes to different outcome measures. For instance, it is clear from the FACET study and other studies that, once a patient with asthma is taking inhaled corticosteroids at $400 \mu \mathrm{g} /$ day or above, the mean improvement in pulmonary function gained by increasing inhaled corticosteroid dose is small.$\left.^{1}\right|^{12}$ However, there may be a significant improvement in terms of prevention of asthma exacerbations when inhaled steroid doses are increased. Addition of a long acting $\beta_{2}$ agonist leads to a more marked improvement in pulmonary function and symptoms and a lesser reduction in asthma exacerbations. 1 12 Addition of a leukotriene receptor antagonist seems both to improve pulmonary function and reduce asthma exacerbations, although the magnitude of this effect compared with other treatments is not yet established. ${ }^{13}$ Theophyllines would appear at present to produce a modest improvement in lung function with, as yet, no evidence of any alteration in asthma exacerbation rate.112 The diverse way in which these different treatments, when added to low dose inhaled corticosteroids, affect asthma outcomes produces difficulties for clinical decision making. It is relatively easy to determine that a drug has improved pulmonary function or decreased symptoms within a short period of time but it is much more difficult to determine that a drug has reduced by $50 \%$ the risk of an asthma exacerbation when a patient may only have one or two asthma exacerbations per year. It is also important to recognise that most clinical trials - and therefore the recommendations of guidelines on which they are based - look at mean results when comparing treatments. It is evident, however, from the FACET study that not all patients experience asthma exacerbations in the same way. Furthermore, as the variability in response to different drugs within clinical trials becomes apparent, it is evident that all patients need to be treated individually when deciding on treatment options and that no one drug or combination of drugs will be suitable for all patients 16

\section{Implications for clinical research}

The trend in recent years to evaluate a number of different outcome measures in clinical trials needs to continue as the true clinical profile of an anti-asthma drug can only be determined by evaluating several different outcome measures. This has implications for the type of clinical trial which needs to be performed; short term clinical trials with relatively limited numbers of patients may be sufficient to determine efficacy using pulmonary function but are unlikely to be of use when measuring asthma exacerbations where longer trials with larger numbers of patients are needed. The desire to evaluate a range of outcome measures may also have implications for the power calculations traditionally done before the start of a trial. These power calculations are usually performed with reference to the primary outcome measure, usually pulmonary function. However, if other outcome measures - particularly asthma exacerbations - are to be measured, then power calculations may have to be performed for each of the important outcome measures in the trial and the number of patients chosen so that all of these outcome measures can be evaluated in a statistically robust fashion. Further research needs to be performed to determine the interaction between different outcome measures. This may eventually enable clinicians to look at some combination of pulmonary function, symptoms, and rescue medication use which indicates a degree of clinical stability $\overrightarrow{\vec{\omega}}$ that makes an asthma exacerbation less likely. It is clear $\overrightarrow{0}$ that pulmonary function alone cannot correlate strongly $\frac{\bar{\sigma}}{\sigma}$ with the risk of asthma exacerbations as, in the FACET $\overline{\bar{p}}$ study, the group on low dose budesonide plus formoterol $\mathbb{\Phi}$ had better lung function than the group on high dose budesonide alone but a greater risk of asthma ex- $~$ acerbation 7 It is evident from the analysis of ex- $\vec{\circ}$ acerbations in the FACET study that not all patients $\overrightarrow{\vec{\omega}}$ behave in the same manner, or at different times the $\stackrel{\sigma}{\sigma}$ same patient may have a different pattern of response. $\overrightarrow{\overrightarrow{5}}$ This needs to be recognised when interpreting and presenting the results of clinical trials, with a move away ir from simply presenting mean data towards showing the of mean data and the individual patient responses. The $\frac{\text { ? }}{0}$ paper by Reddel et $a l^{9}$ shows that outcome measures 0 do not have the same meaning when a patient is im- $-\overrightarrow{.}$ proving as when they are deteriorating. In this study is the improvement in diurnal variation on increase in treatment was a sensitive measure of change. However, 을 increased diurnal variation was not a feature of de- $\vec{\nabla}$ teriorating asthma and would therefore not have been a sensitive measure for worsening of the disease. Furthermore, a decrease in usage of rescue $\beta_{2}$ agonist of four puffs/day when asthma improves may not mean 8 the same as an increase in use of four puffs/day when asthma has previously been well controlled.

\section{Implications for basic research}

It is easy to measure pulmonary function and symptoms $\stackrel{\mathbb{2}}{2}$ but determining the risk of an asthma exacerbation is $\overrightarrow{\overrightarrow{0}}$ difficult. If a simple way could be found to establish 3 that airway inflammation is fully controlled, then it could be possible to determine rationally the dose of anti-inflammatory treatment needed to minimise the risk of exacerbation. The possibility of this approach $\frac{0}{2}$ being successful is suggested by a study in which the $\underset{x}{*}$ measurement of bronchial hyperresponsiveness was 3 added to conventional clinical monitoring of disease to determine treatment needed to improve disease con- 3 trol ${ }^{17}$ However, for use in primary care the measure of bronchial hyperresponsiveness is impractical and some simpler measure is needed. Measurement of exhaled nitric oxide (NO) does not seem to be a suitable candidat 18 and other exhaled measurements are now being o investigated. Another challenge for basic research is to $\mathrm{O}$ try to determine the differing genetic or phenotypic N patterns which underlie variability and response to disease, and a recently reported study indicating a link 0 between polymorphisms in the promoter region of the 5-lipoxygenase gene and response to a leukotriene syn-? thesis inhibitor is of particular interest ${ }^{19}$

\section{Conclusion}

The quality of asthma management has benefited from a clear exposition of the aims of management in various 8 asthma guidelines. It is clear, however, that the relationship between these aims of management such as improvement of pulmonary function, improvement in symptoms, and decrease in the risk of asthma exacerbations is complex and different drugs may have different impacts on these different aims of asthma management. In order to improve our understanding 


\section{LEARNING POINTS}

* The aims of asthma management set out in guidelines are increasingly being reflected in outcome measures used in clinical trials

* To assess some of these outcome measures, particularly asthma exacerbations, will require clinical trials which are longer in duration than has previously been the norm

* There is no universally agreed definition of asthma exacerbations for use in clinical trials

* The relation between different outcome measures in asthma is complex and at present ill understood

* The relation between different outcome measures may vary according to whether asthma is well or poorly controlled and varies between patients

* Despite the availability of carefully measured peak expiratory flow rate in clinical trials, physicians' decision making is still, to a large extent, driven by symptomatology

of the basic pathophysiology of asthma and how it relates to clinical symptoms and lung function, and to improve the stability of health care practitioners to make rational choices about anti-asthmatic drugs, further research is needed into the interrelationship between these different aims of management. The availability of large databases for long term clinical trials provides an excellent opportunity for this research.

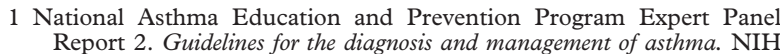
Publication No. 97-4051. Bethesda, Maryland: NHLBI, 1997.

2 British Thoracic Society, The National Asthma Campaign, and The Royal College of Physicians of London in association with The General Practitioner in Asthma Group, The British Association of Accident and Emergency Medicine, The British Paediatric Respiratory Society and The Royal College of Paediatric and Child Health. The British guidelines on asthma management: 1995 review and position stateguidelines on asthma management: 1995

3 Global Initiative for Asthma (GINA). Asthma management and prevention. NIH Publication No. 96-3659A. Bethesda, Maryland: NHLBI, 1995.

4 British Thoracic Society. Guidelines on the management of asthma. Thorax 1993;48:S1-24.

5 Asthma outcome measures. Am F Respir Crit Care Med 1994;149:S1-90.

6 Guidelines for investigating new drugs in asthma. F Soc Pharmaceutical Med 1993.

7 Pauwels RA, Lofdahl C-G, Postma DS, et al on behalf of the FACET International Study Group. Additive effects of inhaled formoterol and budesonide in reducing asthma exacerbations: a one-year, controlled study. N Engl f Med 1997;337:1405-11.
8 Tattersfield AE, Postma DS, Barnes PJ, et al on behalf of the FACET International Study Group. Exacerbations of asthma: a description study of 425 severe exacerbations. Am $\mathcal{F}$ Respir Crit Care Med 1999; 음 160:594-9.

9 Reddel H, Ware S, Marks G, et al. Differences between asthma exacerbations and poor asthma control. Lancet 1999;353:364-9.

10 Gruberg K, Timmers MC, de Klerk EP, et al. Experimental rhinovirus 16 causes variable airflow obstruction in subjects with atopic asthma Am 7 Respir Crit Care Med 1999;160:1375-80.

11 Greening AP, Ind PW, Northfield $M$, et al. Added salmeterol versus higher-dose corticosteroid in asthma patients with symptoms on existing inhaled corticosteroid. Lancet 1994;344:219-24.

12 Woolcock A, Landback R, Ringdal N, et al. Comparison of addition of salmeterol to inhaled steroids with doubling of the dose of inhaled salmeterol to inhaled steroids with doubling of the dose of inhaled

Improved asthma control over 6 weeks with zafirlukast in patients on high dose inhaled corticosteroids. Eur Respir 7 1998:10(Suppl 25): 4375 (abstract).

14 Evans DI. Taylor DA Zetterstrom O, et al. A comparison of lowdose inhaled budesonide plus theophylline and high-dose inhaled budesonide for moderate psthma. N Engl f Med 1997;337:1412-8.

15 Ukena D, Harnest U, Sakalauskas R, et al. Comparison of addition of theophylline to inhaled steroid with doubling of the dose of inhaled steroid in asthm. Eur Respir 7 1998;10:2754-60.

16 Malmstrom K, Rodriguez-Gomez G, Guerra J, et al. Oral montelukast, inhaled beclomethasone and placebo for chronic asthma, a randomised, controlled trial. Ann Intern Med 1999;130:487-95.

7 Sont JK, Willem LN, Bel EH, et al. Clinical control and histopathologic control of asthma under using airway hyperresponsiveness as an additional guide to long-term treatment. Am F Respir Crit Care Med 1999;59:1043-51.

18 Berlyne GS, Barnes NC. No role for NO in asthma. Lancet 2000 (in

press).
19 Ptrogen JM, Yandava CN, Dube L, et al. Pharmacogenetic association between ALOx 5 promoter genotype and the response to anti asthma treatment. Nature Genet 1999;22:168-9. 